\title{
Spain and Chile chosen to host y-ray telescope
}

Sites picked for Cherenkov Telescope Array, which will search for high-energy particles arriving from space.

\section{Chris Cesare}

16 July 2015

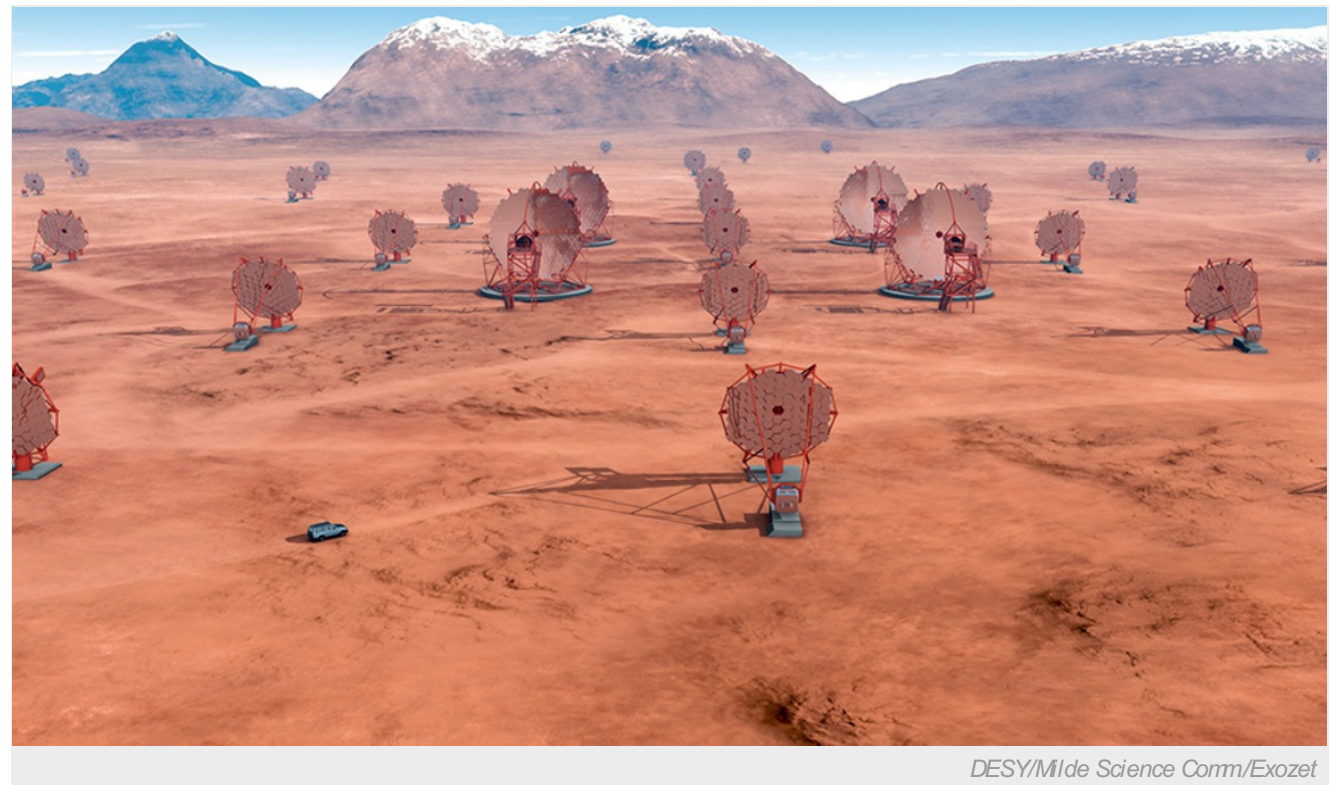

The Cherenkov Telescope Array (artist's impression) will be split across the Northern and Southern hemispheres.

A planned $€ 300$-million (US $\$ 327$-million) telescope array that will try to spot very-high-energy particles streaming from some of the Universe's most cataclysmic events now has two homes.

The Cherenkov Telescope Array (CTA), a collaboration between more than 1,000 scientists from 31 countries, will consist of around 100 dishes in Paranal, Chile, on the grounds of the European Southern Observatory, and around 20 more in La Palma, Spain at the Roque de los Muchachos Observatory. Arrays in both the Northern and Southern hemispheres will provide good coverage of the sky, according to a 16 July press release announcing the decision by the CTA's governing board.

"Because it's looking at particles that are at extremely high energies, it can do similar searches as other high-energy experiments," says Rene Ong, a physicist at the University of California, Los Angeles, and a CTA spokesman. "It's sort of like using the Universe as a laboratory."

The telescope will be watching out for $y$-rays, very-high-energy photons ejected when stars explode or when particles on the edge of black holes accelerate to enormous speeds. When those photons reach Earth's upper atmosphere, they trigger particle showers detectable by ground-based telescopes. The array may also help to probe theories of dark matter, the mysterious stuff that accounts for most of the mass in the Universe but has not yet been seen.

The CTA governing board will now begin to negotiate the detailed contracts ahead of the array's final design and construction, which Ong says should take 3-6 months. It rejected an alternative Southern Hemisphere site in Namibia, while in the Northern Hemisphere, the European site won over competition from Mexico. The time taken to make that decision has seen the telescope's starting construction date slip from the end of 2015 to the middle of 2016.

Nature | doi:10.1038/nature.2015.18008 